Claus C. Schnorrenberger sich bei uns im Westen mit dem Vorwurf konfrontiert, sie sei «unwissenschaftlich». Dieser Vorwurf wird von orthodoxen Schulmedizinern, medizinischen Statistikern, Krankenkassen und manchen Politikern erhoben, ohne dass solche Kritiker die chinesische Heilkunde studiert, geschweige denn sie verstanden hätten. Deren Kritik mag nach den Vorstellungen der Alten Physik sogar gerechtfertigt sein, sie klammert sich aber an obsolete Vorstellungen vom Leben und von der Welt als etwas Objektivem und Materiellem. Nach den Erkenntnissen der Neuen Physik (Quantenphysik) ist sie ein auf mangelnder Information beruhender Irrtum, also eine Täuschung. Die ungelöste Spannung zwischen diesen beiden Polaritäten begleitet den modernen Menschen auf Schritt und Tritt.

Die Neue Physik ist inzwischen über 100 Jahre alt, aber kaum jemand, ausser speziell mit ihr befassten Physikern, hat sie annähernd verstanden. Dabei ist sie wissenschaftlich eindeutig bewiesen, durch Nobelpreise honoriert und unwiderlegbar geworden. Niemand kann in der heutigen Welt leben, ohne die Neue Physik täglich zu benutzen: per Computer, Transistoren, Mobiltelefonen, Fernsehen, Radio und anderen mehr. Der moderne Mensch hantiert gedankenlos mit den entsprechenden Geräten und Medien herum, ohne ihren wissenschaftlichen Hintergrund im Geringsten einsehen zu können.

Die Neue Physik entspricht der Logik der Natur, während die Alte Physik der Logik eines theoretischen Gedankengebäudes, bestehend aus objektivierten Fakten, folgt. Die Alte

\title{
Neue Physik (Quantenphysik) und chinesische Medizin - Teil 1
}

Physik wird neben der Neuen Physik deshalb nicht etwa bedeutungslos; die antike Geometrie eines Euklid, die Erkenntnisse eines Galileo Galilei und Newtons Gesetze gelten weiterhin, doch eröffnet die Neue Physik dem menschlichen Verständnis und Handeln neue Wege und wesentlich weitere Räume.

Interessanterweise enthält die Traditionelle Chinesische Medizin seit über 2000 Jahren Ansätze, die der Neuen Physik näher zu sein scheinen als der abendländischen Alten Physik und die mit der orthodoxen westlichen Medizin unerklärlich sind. Auf solchen Ansätzen beruhen die besonderen Wirkungen der chinesischen Medizin einschliesslich der Akupunktur, die den Möglichkeiten der modernen westlichen Heilkunde oftmals klar überlegen sind. Dies haben intelligente Zeitgenossen akzeptiert, denn der Zustrom von Patienten zur chinesischen Medizin ist im Abendland sowie in Asien, Afrika und Australien ungebrochen, obwohl die Behandlung von Krankenkassen meist nicht honoriert wird. Mit einigen Hintergründen dazu befasst sich der vorliegende Beitrag.

\section{Neue Physik}

Die Neue Physik (Quantenphysik) erklärt uns: Materie ist nicht aus Materie aufgebaut. Das primäre Phänomen ist Beziehung; Materie ist nur das sekundäre Resultat. Materie ist kristallisierte Form [1-5]. Am Ende der Teilung von Materie in kleine und immer kleinere Teilchen bleibt etwas übrig, das dem Geistigen ähnelt: Es ist ganzheitlich, offen und lebendig; es ist Potenzialität, die Kann-Möglichkeit einer Realisierung. Materie ist nur die Schlacke dieses Geistigen - zerlegbar, abgrenzbar, determiniert: Realität. Im Grunde gibt es nur Geist.

Doch dieser Geist «verkalkt», und er wird, wenn er verkalkt, zu Materie. In der Quanten-Welt gibt es so etwas wie Materie überhaupt nicht. Materie bildet sich erst als «Als ob»-Erscheinung bei grösserer Anhäufung atomarer Gestaltwesen auf einem räumlich höheren Niveau heraus. Materie ist somit für die Quantenphysik ein «illusionäres» Phänomen, etwas, das die volle Realität der Welt verschleiert. Die deutsche Sprache nennt Realität «Wirklichkeit»; das ist etwas, das arbeitet, sich bewegt, wirkt und lebt [6].

Materie ist wie der geschlossene Vorhang vor einer Theaterbühne, auf der die wirklichen Dinge dahinter passieren. So gesehen ist das «Objektivierte» in der Betrachtungsweise der westlichen Wissenschaft, d.h. der Alten Physik, eine Verschleierung der eigentlichen, d.h. der ganzheitlichen und vollen Wirklichkeit.

Werden die üblichen Diagnosen der orthodoxen westlichen Medizin unter solchen Aspekten betrachtet, müssen sie einem wie Maskeraden vorkommen: Sie verschleiern die medizinische Wirklichkeit mit Wortattrappen, die aus der Sicht des Ganzen bedeutsamere innere Abläufe sowie wirksamere und pathophysiologische Veränderungen im Menschen verhüllen und verdrängen. Solche Veränderungen und Abläufe stehen unter anderem im Mittelpunkt der sogenannten chinesischen SyndromDiagnosen (Bian-Zheng 辨證). Die orthodoxe westliche Schulmedizin bezeichnet diese Syndrome aus purer

\section{KARGER}

Fax +4976145207 14

Information@Karger.com

www.karger.com
() 2014 S. Karger GmbH, Freiburg

Accessible online at: www.karger.com/szg
Prof. h.c. (China Medical University, Taichung, Taiwan, Rep. China)

Dr. med. Claus C. Schnorrenberger, M.D.

Lifu International College of Chinese Medicine

Karl Jaspers-Allee 8, 4052 Basel, Schweiz

lifu@gmx.ch

www.lifu-chinesischemedizin.ch 
Ignoranz als unwissenschaftlich. Der differenzierte Zeitablauf des inneren Gleichgewichts, der für Chinas Medizin entscheidend ist, bleibt bei den starren westlichen Diagnosen unbeachtet auf der Strecke. Er wird von orthodoxen Medizinern ignoriert, mystifiziert oder einfach als unsinnig dargestellt.

Dem chinesischen Arzt erscheinen demgegenüber viele westliche Diagnosen wie oberflächliche Täuschungen. Beispiele dafür sind: Hepatitis, Gastritis, Bandscheibenvorfälle, Diabetes mellitus, Migräne usw., die mittels chinesischer Diagnostik und Therapie kritisch analysiert, pathophysiologisch völlig enthüllt und ganzheitlich richtig behandelt werden können. Warum ist das so?

Die Logik der Natur, der die Neue Physik folgt und die ein guter Arzt kennen sollte, bleibt bei den meisten westlichen Diagnosen unberücksichtigt. Deshalb können viele Krankheiten nicht mit westlicher Medizin, und sei sie technologisch noch so perfekt ausgestattet, adäquat diagnostiziert und erfolgreich angegangen werden, und deshalb gibt es in der westlichen Medizin unerwünschte medizinische Nebenwirkungen. Die moderne Pharmakologie behauptet zwar, es gäbe keine medizinische Wirkung ohne Nebenwirkung, doch ist dies nur so, weil der unzulängliche Ansatz der orthodoxen westlichen Heilkunde, der die natürliche Logik des menschlichen Organismus nicht verstehen kann, zugrunde gelegt wird. Hier liegt die Domäne der chinesischen Medizin, die nahezu nebenwirkungsfrei ist, und dies deshalb, weil sie näher an der Neuen Physik, der Quantenphysik, ist als die orthodoxe Heilkunde. Damit ist sie näher an der Wirklichkeit, denn sie entspricht der Logik der Natur.

Die kleinsten Teilchen der Neuen Physik sind viel kleiner als Atome, Atomkerne, Elektronen und Neutronen. Solche Teilchen sind, wie gesagt, nicht materiell: Sie arbeiten, sie bewe- gen sich, sie leben. Diese kleinsten Teilchen können nach Professor Hans Peter Dürr als «happenings» beschrieben werden; sie sind winzige Artikulationen der Wirklichkeit [6]. Sie sind ein Feld von Information, das nicht in Materie und Energie aufgespalten ist. Dieses Feld von Information besteht nicht bloss innerhalb eines jeden lebenden Wesens, es reicht so weit wie der gesamte Kosmos. Dieser Kosmos ist ein Gesamt-Ganzes. In der chinesischen Tradition wird das mittels SHEN 神 (Lingshu Jing, Kapitel 1) und $D A O$ 道 ausgedrückt [7].

Die Quantenphysik sagt: Die Welt ist ein Ganzes, und ihre Realität (d.h. ihre Wirklichkeit) ist eine unendliche spirituelle Verbindung. In der subatomaren Welt der Quanten gibt es keine Objekte, keine materiellen Dinge, keine Substanzen; in ihrer Sprache gibt es keine Substantive. Es gibt nur Bewegung, Prozesse, Verbindungen und Information. Das volle Verständnis der Quantenphysik enthält keine Möglichkeit, die Welt in lauter Teilchen zu zerlegen. Denn die kleinsten subatomaren Teilchen verhalten sich wie Wellen, und diese Wellen verhalten sich wie Teilchen. Es ist kein scharfer Unterschied zwischen beiden feststellbar.

Dieses dualistische Prinzip wurde 1927 von Werner Heisenberg als «Unschärferelation» (englisch «uncertainty» oder «blur relation») bezeichnet, und eben diese Unschärfe zeigt den Ursprung des Lebendigen an. Es ist ein universeller Code, der auf Information beruht $[4,6]$. Das hat eine Parallele zum dualistischen Yin-Yang 陰陽, dem Prinzip der chinesischen Tradition.

Wir finden eine solche Unschärfe in der Struktur der chinesischen medizinischen Syndrome (Bian-Zheng 辨 證), wo ein Syndrom durch ein anderes ersetzt werden kann oder mehrere Syndrome gleichzeitig aktiv sein können; das ist nicht «exakt» oder «genau", eben weil es auf dem Ganzen beruht. Wir werden im Folgenden einige solcher Syndrome im Bezug zu entsprechenden westlichen Diagnosen analysieren.

Sicherheit (engl. certainty) kann in eine Bian-Zheng-Diagnose nur sekundär hineinprojiziert werden, was in der chinesischen Medizin schon im Altertum durch induktive bzw. akzidentielle Strategien wie unter anderem Anatomie, Embryologie, Kräuterrezepturen, Akupunkturtechniken sowie Zungen- und Pulsdiagnostik geschehen ist und was in unserer Zeit zusätzlich durch moderne Technologien, aber auch durch Methoden der orthodoxen Medizin, die durchaus brauchbar und oft sogar notwendig sind, erfolgt. Es wird heute ausserdem mit scheinbar wissenschaftlichen Untersuchungen nach der sogenannten «Evidence-Based Medicine» (EBM) gesucht, wobei der Nachteil der Vernachlässigung des lebendigen Ganzen als logischer Grundfehler stillschweigend in Kauf genommen wird. Die EBM beruht auf einer ganzen Reihe logischer Irrtümer, was ich in meinem Beitrag «Wissenschaftstheoretische Bewertung der chinesischen Medizin und Akupunktur» $[8,9]$ eingehend dargestellt habe.

Der grundlegende Welle-TeilchenDualismus zeigt, dass es unsinnig ist, die Welt rein "physikalisch» erklären zu wollen, indem man sie auf materielle Dinge, auf Bewusstsein oder auf Energie reduziert.

Realität (im Deutschen: Wirklichkeit) ist im Verständnis der Quantenphysik keine materielle Sache; es ist, wie gesagt, Potenzialität, Möglichkeit. Materie ist somit eine Art Zaubertrick, der in unserer begrenzten menschlichen Wahrnehmung begründet ist. (Letzteres ähnelt der buddhistischen Lehre von MAYA.) Denn unser modernes menschliches Gehirn ist nicht darauf trainiert, die Neue Physik unmittelbar zu verstehen.

Wir halten fest: Es gibt nur ein einheitliches Ganzes. Mensch und Natur sind nicht voneinander getrennt; beide sind vielmehr ein einheitliches Ganzes. 
Wir finden eine ähnliche Auffassung in Aristoteles' berühmtem Buch über die Physik ( $\ \Upsilon \Sigma \mathrm{IKH} \Sigma$ $\operatorname{AKPOA} \Sigma \mathrm{E} \Omega \Sigma$ ) [10], das zum Ausgangspunkt aller späteren logischen Folgerungen und wissenschaftlichen Errungenschaften des Abendlandes geworden ist. Aristoteles nennt die

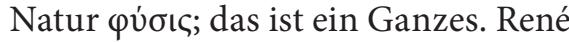
Descartes versuchte im 17. Jahrhundert, die Welt willkürlich in Materie und Bewusstsein zu zerlegen - ein Irrtum, bei dem der Philosoph einer vordergründigen Genauigkeit nachjagte, die es in der Natur gar nicht gibt, deren Gespenst aber in den Köpfen vieler moderner Menschen weiterhin verwirrend herumspukt. Es gibt heute keinen wissenschaftlichen Grund mehr für eine Erklärung der Welt durch Reduktion auf Materie oder auf materielle Dinge. Das wäre Alte Physik. Die Logik der Quantenphysik, d.h. der Neuen Physik, ist dagegen die Logik der Natur.

\section{Chinesische Medizin}

Wenden wir uns der Lehre der chinesischen Medizin zu: Im 41. Kapitel des Buches Lingshu Jing, «YIN-YANG - SONNE und MOND», wird der Mensch einschliesslich seiner Blutgefässe (Jing-Mai 經脈), inneren Organe (Wu-Zang Liu-Fu 五藏 六腑) und Akupunkturstrukturen (Xue-Wei 穴 位) als integrierter Teil des Kosmos analysiert. Nach dieser Ansicht beruhen die Welt und das menschliche Leben auf den 12 Erdzweigen und 10 Himmelsstämmen, was auf das YI JING 易經 (Buch der Wandlungen) und eine alte Erklärung der Welt gemäss Tian - Ren - Di 天人地 («Der Mensch steht mitten zwischen Himmel und Erde») zurückgeht. Letzteres wurde bei der Erklärung der chinesischen Medizin im Westen bisher ignoriert. Man redet dort von «Meridianen», von «Punkten» und von «Energie», die es in den originalen
Texten nie gegeben hat. Im kommunistischen China der Volksrepublik verordnete Mao Zedong der chinesischen Medizin zu allem Überfluss eine dialektisch-materialistische Rosskur, bei der viele der alten «transzendenten» Erkenntnisse und Erfahrungen unterdrückt wurden, die erst allmählich im Reich der Mitte wieder entdeckt werden. In der kommunistischen Volksrepublik China geschulte westliche Akupunkteure erhielten so ein falsches Bild von Chinas originaler Heilkunde verpasst, was in westlichen Zirkeln eifrig verbreitet worden ist. Das führte zu einer verzerrten Interpretation der chinesischen Medizin und Akupunktur im Westen. Dabei ist die originale chinesische Medizin höchst differenziert, und im Lichte der Quantenphysik ist sie auch durchaus wissenschaftlich (Tab. 1-7).

Die Bahnen der chinesischen Medizin und Akupunktur sind nach der universellen kosmischen Ordnung

Tab. 1. Die 10 Himmelsstämme (Tian Gan 天干) (nach dem YI JING 易經)

\begin{tabular}{|c|c|c|c|c|c|c|c|c|c|}
\hline 1 & 2 & 3 & 4 & 5 & 6 & 7 & 8 & 9 & 10 \\
\hline Jia 甲 & Yi 乙 & Bing 丙 & Ding 丁 & $\mathrm{Wu}$ 戊 & Ji 己 & Geng 庚 & Xin 辛 & Ren 1 & Gui 癸 \\
\hline
\end{tabular}

Tab. 2. Die 12 Erdzweige (Di Zhi 地支) (nach dem YI JING 易經)

\begin{tabular}{|c|c|c|c|c|c|c|c|c|c|c|c|}
\hline 1 & 2 & 3 & 4 & 5 & 6 & 7 & 8 & 9 & 10 & 11 & 12 \\
\hline $\mathrm{Zi}$ 子 & Chou \# & Yin 寅 & Mao 卯 & Chen 辰 & Si e & $\mathrm{Wu}$ 午 & Wei 未 & Shen 申 & You 酉 & $\mathrm{Xu}$ 酉 & Hai 亥 \\
\hline
\end{tabular}

Tab. 3. Einteilung des Tages in 12 Doppelstunden nach den 12 Erdzweigen

\begin{tabular}{|c|c|c|c|c|c|c|c|c|c|c|c|c|}
\hline Stunden & $\mathrm{Zi}$ & Chou & Yin & Mao & Chen & $\mathrm{Si}$ & $\mathrm{Wu}$ & Wei & Shen & You & $\mathrm{Xu}$ & Hai \\
\hline Tageszeit & $23-1$ & $1-3$ & $3-5$ & $5-7$ & $7-9$ & $9-11$ & $11-13$ & $13-15$ & $15-17$ & $17-19$ & $19-21$ & $21-23$ \\
\hline
\end{tabular}

Tab. 4. Die 5 Transportpunkte (Shu-Xue 输穴) der 5 Speicherorgane in Bezug zu den Jahreszeiten, Farben, Tönen und Geschmack

\begin{tabular}{|c|c|c|c|c|c|c|c|}
\hline 5 Veränderungen & $\begin{array}{l}5 \text { Jahres- } \\
\text { zeiten }\end{array}$ & $\begin{array}{l}5 \text { Transport- } \\
\text { punkte }\end{array}$ & Leber & Lunge & Herz & Milz & Niere \\
\hline Verbergen & Winter & Jing 井 & $\begin{array}{l}1 \\
\text { Da Dun }\end{array}$ & $\begin{array}{l}11 \\
\text { Shao Shang }\end{array}$ & $\begin{array}{l}9 \\
\text { Shao Chong }\end{array}$ & $\begin{array}{l}1 \\
\text { Yin Bai }\end{array}$ & $\begin{array}{l}1 \\
\text { Yong Quan }\end{array}$ \\
\hline Farben & Frühling & Rong 榮 & $\begin{array}{l}2 \\
\text { Xing Jian }\end{array}$ & $\begin{array}{l}10 \\
\text { Yu Ji }\end{array}$ & $\begin{array}{l}8 \\
\text { Shao Fu }\end{array}$ & $\begin{array}{l}2 \\
\mathrm{Da} \mathrm{Du}\end{array}$ & $\begin{array}{l}2 \\
\operatorname{Ran} \mathrm{Gu}\end{array}$ \\
\hline 5 Jahreszeiten & Sommer & Shu 腧 & $\begin{array}{l}3 \\
\text { Tai Chong }\end{array}$ & $\begin{array}{l}9 \\
\text { Tai Yuan }\end{array}$ & $\begin{array}{l}7 \\
\text { Shen Men }\end{array}$ & $\begin{array}{l}3 \\
\text { Tai Bai }\end{array}$ & $\begin{array}{l}3 \\
\text { Tai Xi }\end{array}$ \\
\hline Töne & Spätsommer & Jing 經 & $\begin{array}{l}4 \\
\text { Zhong Feng }\end{array}$ & $\begin{array}{l}8 \\
\text { Jing Qu }\end{array}$ & $\begin{array}{l}4 \\
\text { Ling Dao }\end{array}$ & $\begin{array}{l}5 \\
\text { Shang Qiu }\end{array}$ & $\begin{array}{l}7 \\
\text { Fu Liu }\end{array}$ \\
\hline Geschmack & Herbst & $\mathrm{He}$ 合 & $\begin{array}{l}8 \\
\text { Qu Quan }\end{array}$ & $\begin{array}{l}5 \\
\text { Chi Ze }\end{array}$ & $\begin{array}{l}3 \\
\text { Shao Hai }\end{array}$ & $\begin{array}{l}9 \\
\text { Yin Ling Quan }\end{array}$ & $\begin{array}{l}10 \\
\text { Yin Gu }\end{array}$ \\
\hline
\end{tabular}


Tab. 5. Die 12 Gefässe der Akupunktur, die 12 Monate und die 12 Erdzweige $^{\mathrm{a}}$

\begin{tabular}{|c|c|c|c|c|}
\hline Monat & Gefäss & Verbunden mit & Monat & Gefäss \\
\hline Oktober & $\begin{array}{l}\text { Zu Jue Yin (links) } \\
\text { Leber }\end{array}$ & Hai - Xu & September & $\begin{array}{l}\text { Zu Jue Yin (rechts) } \\
\text { Leber }\end{array}$ \\
\hline November & $\begin{array}{l}\text { Zu Tai Yin (links) } \\
\text { Milz }\end{array}$ & $\mathrm{Zi}-\mathrm{You}$ & August & $\begin{array}{l}\text { Zu Tai Yin (rechts) } \\
\text { Milz }\end{array}$ \\
\hline Dezember & $\begin{array}{l}\text { Zu Shao Yin (links) } \\
\text { Niere }\end{array}$ & Chou - Shen & Juli & $\begin{array}{l}\text { Zu Shao Yin (rechts) } \\
\text { Niere }\end{array}$ \\
\hline Januar & $\begin{array}{l}\text { Zu Shao Yang (links) } \\
\text { Gallenblase }\end{array}$ & Yin - Wei & Juni & $\begin{array}{l}\text { Zu Shao Yang (rechts) } \\
\text { Gallenblase }\end{array}$ \\
\hline Februar & $\begin{array}{l}\text { Zu Tai Yang (links) } \\
\text { Blase }\end{array}$ & $\mathrm{Mao}-\mathrm{Wu}$ & Mai & $\begin{array}{l}\text { Zu Tai Yang (rechts) } \\
\text { Blase }\end{array}$ \\
\hline März & $\begin{array}{l}\text { Zu Yang Ming (links) } \\
\text { Magen }\end{array}$ & Chen - Xu & April & $\begin{array}{l}\text { Zu Yang Ming (rechts) } \\
\text { Magen }\end{array}$ \\
\hline
\end{tabular}

${ }^{a}$ Die Gefässbahnen der Akupunktur (chin. Jing-Mai 經脉), im Westen irrtümlich «Meridiane» genannt, leiten sich also direkt aus der kosmischen Yin-YangOrdnung her.

Tab. 6. Die Himmelsstämme und die Gefässe der Hand

\begin{tabular}{|c|c|c|c|c|c|}
\hline Nummer & $\begin{array}{l}\text { Himmels- } \\
\text { stamm }\end{array}$ & Gefäss & Nummer & $\begin{array}{l}\text { Himmels- } \\
\text { stamm }\end{array}$ & Gefäss \\
\hline 9 & Ren & $\begin{array}{l}\text { Shou Tai Yin (links) } \\
\text { Lunge }\end{array}$ & 8 & Xin & $\begin{array}{l}\text { Shou Tai Yin (rechts) } \\
\text { Lunge }\end{array}$ \\
\hline 10 & Gui & $\begin{array}{l}\text { Shou Shao Yin (links) } \\
\text { Herz }\end{array}$ & 7 & Geng & $\begin{array}{l}\text { Shou Shao Yin (rechts) } \\
\text { Herz }\end{array}$ \\
\hline 1 & Jia & $\begin{array}{l}\text { Shou Shao Yang (links) } \\
\text { Drei Erwärmer }\end{array}$ & 6 & $\mathrm{Ji}$ & $\begin{array}{l}\text { Shou Shao Yang (rechts) } \\
\text { Drei Erwärmer }\end{array}$ \\
\hline 2 & $\mathrm{Yi}$ & $\begin{array}{l}\text { Shou Tai Yang (links) } \\
\text { Dünndarm }\end{array}$ & 5 & $\mathrm{Wu}$ & $\begin{array}{l}\text { Shou Tai Yang (rechts) } \\
\text { Dünndarm }\end{array}$ \\
\hline
\end{tabular}

Tab. 7. Die 5 Speicherorgane im Bezug auf die Fünf Elemente, Farben, Jahreszeiten, Töne, Geschmack und die 10 Himmelsstämme

\begin{tabular}{|c|c|c|c|c|c|}
\hline & \multicolumn{5}{|l|}{ Organe } \\
\hline & Leber & Herz & Milz & Lunge & Niere \\
\hline Fünf Elemente & $\mathrm{Holz}$ & Feuer & Erde & Metall & Wasser \\
\hline Jahreszeit & Frühling & Sommer & Spätsommer & Herbst & Winter \\
\hline Musikalische Töne & Jue 角 & Zhi 徵 & Gong 宮 & Shang 商 & Yu 羽 \\
\hline Geschmack & sauer & bitter & süss & scharf & salzig \\
\hline 10 Himmelsstämme & Jia & Bing & $\mathrm{Wu}$ & Geng & Ren \\
\hline
\end{tabular}

Tab. 8. Ein Vergleich zwischen chinesischen Originalnamen und westlichen Punkt-Nummern

\begin{tabular}{lll}
\hline $\begin{array}{l}\text { Chinesischer } \\
\text { Originalname }\end{array}$ & Übersetzung & $\begin{array}{l}\text { Westliche } \\
\text { Punktnummer }\end{array}$ \\
\hline $\begin{array}{l}\text { Tian Fu 天府 } \\
\text { Shang Yang 商陽 }\end{array}$ & Haus des Himmels & Lunge 3 \\
Shen Men 神門 & Der 4. Ton Shang 商 im YANG & Dickdarm 1 \\
Shen Tang 神堂 & Tor des SHEN & Herz 7 \\
Shen Feng 神封 & Halle des SHEN & Blase 44 \\
Shen Zang 神藏 & Haus des SHEN & Niere 23 \\
Yin Du 陰都 & Speicher des SHEN & Niere 25 \\
Shen Dao 神道 & Versammlung des YIN & Niere 19 \\
Shen Ting 神庭 & Bahn des DAO & Lenkergefäss 11 \\
Qi Hai 氣海 & Halle des SHEN & Lenkergefäss 24 \\
Tian Chi 天池 & Ozean des QI & Konzeptionsgefäss 6 \\
Tian Quan 天泉 & Wasserbecken des Himmels & Perikard 1 \\
Ri Yue 日月 & Quelle des Himmels & Perikard 2 \\
Yang Jiao 陽交 & Sonne-Mond (Yang-Yin) & Gallenblase 24 \\
Yang Fu 陽輔 & Kreuzung des YANG & Gallenblase 35 \\
Yin Gu 陰谷 & Kutsche des YANG & Gallenblase 38 \\
Yin Bao 陰包 & Tal des YIN & Niere 10 \\
Yin Jiao 陰交 & YIN des Uterus & Leber 9 \\
\hline
\end{tabular}

von Yin-Yang 陰陽 benannt, haben aber auch anatomische Bezüge. Sie heissen:

- Tai Yang 太陽,

- Shao Yang 少陽,

- Yang Ming 陽明,

- Tai Yin 太陰,

- Shao Yin 少陰,

- Jue Yin 厥陰.

Zahlreiche Akupunkturpunkte tragen originale Namen, die auf die bipolare kosmische Ordnung des Ganzen gemäss Yin-Yang sowie auf das obige Tian - Ren - Di hinweisen (Tab. 8), wie z.B.: 
- Tian Fu 天府,

- Shen Men 神門,

- Shen Tang 神堂,

- Shen Feng 神封,

- Shen Zang 神藏,

- Shen Dao 神道,

- Shen Ting 神庭,

- Qi Hai 氣海,

- Shang Yang 商陽,

- Yin Du 陰都,

- Tian Chi 天池,

- Tian Quan 天泉,

- Ri Yue 日月,

- Yang Jiao 陽交,

- Yang Fu 陽輔,

- Yin Gu 陰谷,

- Yin Bao 陰包,

- Yin Jiao 陰交 und andere mehr.

Das grundlegende Verständnis für die kosmische Einordnung der chinesischen Foramina verschwindet völlig bei der simplifizierenden westlichen Nummerierung von Akupunktur-Punkten (die eigentlich dreidimensionale Höhlen-Strukturen - chin. Xue-Wei 穴位 - sind). Dies wird bei einem Vergleich mit den im Westen üblichen Abkürzungen deutlich, wobei mit den Kürzeln keinerlei grundlegende Beziehungen erklärt, sondern vielmehr solche Beziehungen zum Ganzen unkenntlich gemacht werden (Tab. 8).

Das chinesische Gefässsystem ist auf solche Beziehungen gegründet, auf die auch die Beziehungen der inneren Organe untereinander zurückgehen und aus denen später die Syndrome (Bian-Zheng 辩證) entstehen. Die Gefässverläufe ermöglichen unter anderem das Verständnis folgender Organbeziehungen:

- Tai Yang: Blase-Niere-DünndarmHerz-Lunge.

- Shao Yang: Dünndarm-Gallenblase-Leber-Drei Erwärmer-MagenPerikard.

- Yang Ming: Magen-DickdarmDünndarm-Milz-Herz.

- Tai Yin: Milz-Magen-Herz-LeberNiere-Dickdarm-Dünndarm-Herz.

- Shao Yin: Herz-Niere-DünndarmBlase-Lunge.
- Jue Yin: Perikard-Drei ErwärmerLeber-Gallenblase-Herz-Magen.

Dies ergibt eine Fülle von Möglichkeiten allein bei der Behandlung mit Akupunktur. Diese Möglichkeiten sind nicht wahllos «erfunden», sondern sie folgen einem vorhersehbaren Plan, wobei das Verständnis für die Einheit des Gefässsystems und des menschlichen Organismus als Teil des Universums, des Kosmos, wesentlich ist. Auf solchen Möglichkeiten beruht die Struktur der Syndrome.

Was in der Quantenphysik «Geist» genannt wird, entspricht in der chinesischen Medizin dem SHEN 神, auf dem die wissenschaftliche Wirklichkeit der chinesischen Heilkunde letztlich beruht [7].

Es ist daher unsinnig und wissenschaftlich gesehen geradezu falsch, die traditionelle chinesische Medizin und die originale Akupunktur auf Begriffe und Theorien der sogenannten orthodoxen westlichen Heilkunde reduzieren $\mathrm{zu}$ wollen, um sie einer intellektuell zurückgebliebenen Allgemeinheit, für die die Alte Physik eine Art Evangelium ist, verständlich $\mathrm{zu}$ machen. Doch wird dieser Unfug heute im Westen in weitem Umfang betrieben, wobei enorme Forschungsgelder sinnlos verschleudert werden.

\section{Weitere Methoden der traditionellen chinesischen \\ Medizin}

Die Fünf Elemente sind eine historisch frühe Vorwegnahme der wechselseitigen Steuerung (Abb. 1), womit

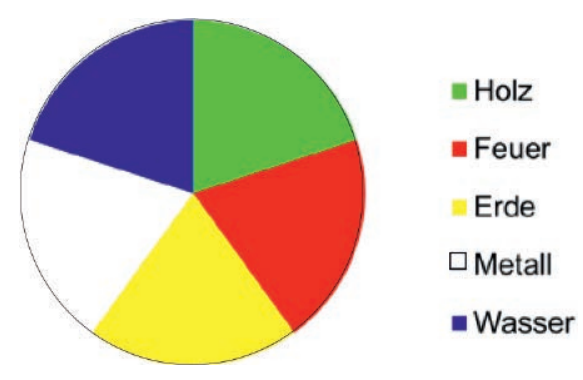

Abb. 1. Die Fünf Elemente mit den zugeordneten 5 Farben.

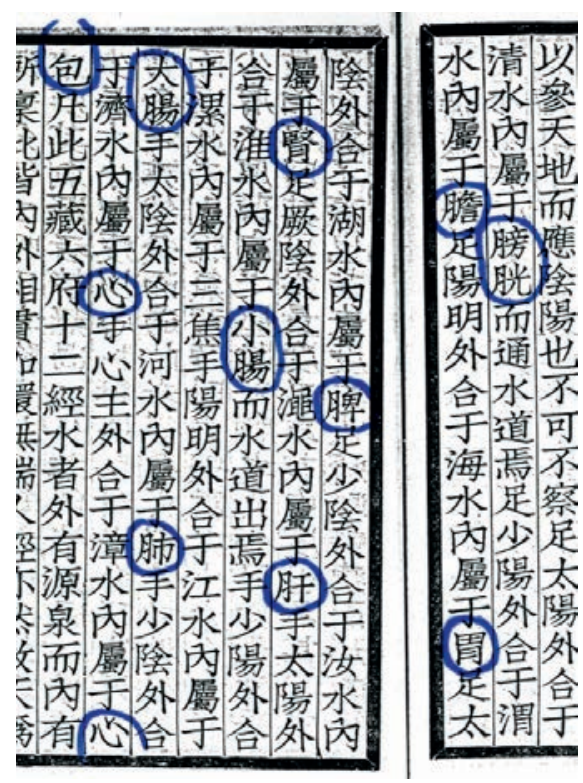

Abb. 2. Namen der inneren Organe.

sich heute die Kybernetik befasst. Die Fünf Musikalischen Töne (sie sind ähnlich der Sphärenmusik des Pythagoras zu verstehen):

- Jue 角,

- Zhi 徵,

- Gong 宫,

- Shang 商,

- $\mathrm{Yu}$ 羽.

Materielle, objektivierbare Aspekte wurden schon früh in die traditionelle chinesische Medizin hineingetragen. Dazu gehören:

- Die frühe chinesische Anatomie: Diese wird in Kapitel 12 des Lingshu Jing Jie-Pou 解剖 genannt. Der Begriff Jie-Pou wird in China noch heute für die moderne medizinische Anatomie verwendet, ebenso wie die Namen für die inneren Organe des Menschen. Darunter ist das Zwerchfell $G e$ 膈, das nur bei der anatomischen Sektion einer Leiche sichtbar wird. Es wird im Buch Lingshu Jing an vielen Stellen als Orientierungsmarke angegeben [11]. Die Namen der inneren Organe, die in der modernen chinesischen Medizin dieselben sind, werden im Kapitel 12 des Lingshu Jing, einem Text, der vor über 2500 Jahren entstand, vollständig aufgeführt (Abb. 2, 3). 


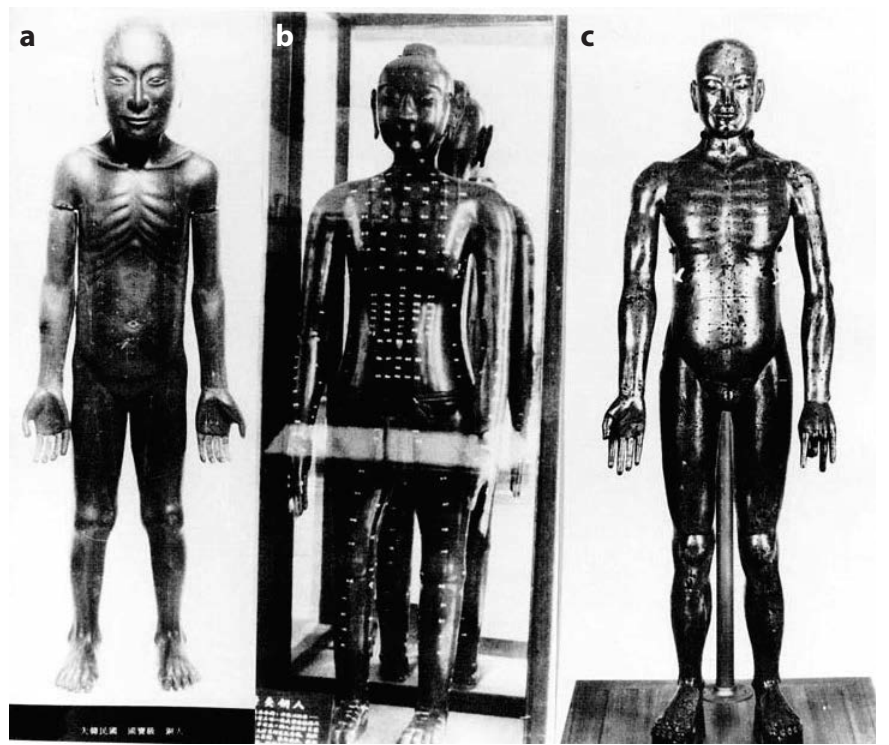

Abb. 3. Der Bronze-Mensch (Kupfer-Mann) der Akupunktur (Tong Ren 銅人) wurde entsprechend modernen anatomischen Standards von Wang Wei-Yi um 1060 n. Chr. geschaffen (nach Prof. Hongchien Ha, M.D.). a Koreanischer Bronze-Mensch. b Bronze-Mensch aus der Ming-Dynastie. c Der originale Bronze-Mensch von 1060, der im kaiserlichen Museum in Tokio aufbewahrt wird.

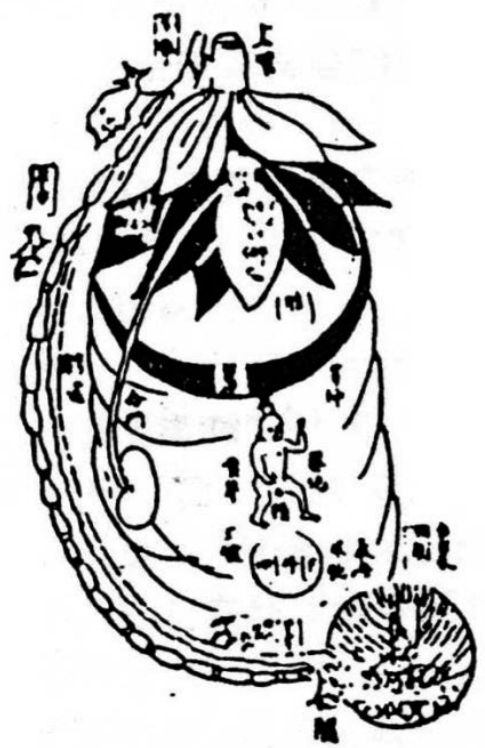

Abb. 4. Chinesische Illustration der Embryonalentwicklung (die unscharfe Wiedergabe einiger Schriftzeichen beruht auf dem ungenügenden Original).
- Eine Embryologie, welche die vorgeburtliche Entwicklung des Menschen richtig beschreibt: Im Kapitel 10 des Lingshu Jing erklärt der chinesische Kaiser die Zeugung des Menschen durch die Kombination einer weiblichen Eizelle Jing 精 (der moderne chinesische Terminus für die weibliche Eizelle ist Luan $\mathrm{Zi}$ 卵子) mit einer männlichen Samenzelle Jing 精. Wenn sich beide vereinigt haben, entwickeln sich zunächst das Gehirn, Nao 腦, und das Rückenmark, Sui 髓 (die beide dem Ektoderm der drei Keimblätter der modernen Embryologie angehören) (Abb. 4).

Dann entstehen die Knochen, $G u$ 骨, und die Blutgefässe $M a i$ 脈, in denen Blut, Xue 血, und $Q i$ 氣 zirku- lieren. Diese Gefässe transportieren Nährstoffe, Ying 營, sowie Agentien der Immunabwehr, Wei 衛. Der Kaiser betont: «Blut und $Q i$ (Xue-Qi 血氣) zirkulieren ständig in den embryonalen Gefässen ohne Anfang und Ende.» (Diese chinesische Quelle ist die erste in der Medizingeschichte, welche die menschliche Blutzirkulation erwähnt, die für den Westen erst im 17. Jahrhundert durch William Harvey entdeckt wurde.) «Danach erscheinen die Sehnen, Jin 筋, und die Muskeln, Rou 肉. Beide schützen die inneren Organe, Zang Fu 臟腑. Schliesslich entstehen die Haut, $P i$ 皮, und die Haare, Mao 毛, und danach ist das neue menschliche Wesen fertig.»

\section{Literatur}

1 Heisenberg W: Quantentheorie und Philosophie. Stuttgart, Reklam Universal-Bibliothek, 1979.

2 Heisenberg W: Das Naturbild der heutigen Physik. Hamburg, Rowohlt, 1955.

3 Heisenberg W: Der Teil und das Ganze. München, Piper, 1969.

4 Heisenberg W: Physics and Philosophy. London, Penguin Books, 1958/1989.

5 Cox B, Forshaw J: The Quantum Universe: Everything that Can Happen Does Happen. London, Penguin Books, 2012.

6 Dürr HP: Das Lebendige lebendiger machen. München, oekom, 2011.

7 Huangdi Neijing Lingshu 黄帝內經靈樞. Ausgabe Peking 靈樞經白話解. Hongkong, Beijing Cultural Publishing House, 1971.

8 Schnorrenberger CC: Wissenschaftstheoretische Bewertung der chinesischen Medizin und Akupunktur, Teil 1. Schweiz Z Ganzheitsmedizin 2011;23:275-284.

9 Schnorrenberger CC: Wissenschaftstheoretische Bewertung der chinesischen Medizin und Akupunktur, Teil 2. Schweiz Z Ganzheitsmedizin 2011;23:332-340.

10 Artistoteles: Physik. Englisch: Lecture on Nature $\Phi \Upsilon \Sigma I K H \Sigma$ AKPOA $\Sigma E \Omega \Sigma$. Cambridge, Mass., und London/England, Harvard University Press, 1929.

11 Schnorrenberger CC: Anatomical roots of acupuncture. Schweiz Z Ganzheitsmedizin 2013;25:110-118. 